\title{
Sensorineural Hearing Loss as a Sequelae of Radiotherapy and Chemotherapy in Head and Neck Cancer - An Observational Study from Maharashtra, India
}

\author{
Sanket Chandrashekhar Prabhune ${ }^{1}$, Abhay D. Havle², Swapna Ajay Shedge 3 , \\ Khaleel Basha Mannuru' ${ }^{4}$ Lakshmi Sravya Yarlagadda ${ }^{5}$, Kaenat Ahmed ${ }^{6}$ \\ 1, 2, 4, 5, 6 Department of ENT, Krishna Institute of Medical Sciences (Deemed to Be \\ University), Karad, Maharashtra, India, ${ }^{3}$ Department of Anatomy, Krishna Institute of \\ Medical Sciences (Deemed to Be University), Karad, Maharashtra, India.
}

\section{ABSTRACT}

\section{BACKGROUND}

Radiotherapy is a very well-known treatment modality for head and neck cancers besides surgery. The cochlea and its neuroepithelium are sensitive to ionizing radiation and resultant damage as it remains in the field of irradiation, the chemotherapy also has a similar effect leading to sensorineural hearing loss (SNHL). To minimize the adverse effects of hearing the advent of technology like intensitymodulated radiotherapy (IMRT) using smaller doses of radiation is now available with good control of the disease. The intended concomitant uses of chemotherapeutic agent cisplatin for increasing the sensitivity of radiation may induce ototoxicity. Both of these modalities result in a pronounced effect on highfrequency sensorineural hearing loss. We wanted to determine and compare sensorineural hearing loss amongst cases of head and neck cancer treated by radiotherapy, chemotherapy either alone or in a combination of both.

\section{METHODS}

All clinically diagnosed patients of head and neck cancer requiring treatment using radiotherapy or chemotherapy alone or in combination having a normal hearing on pure tone audiometry (PTA) were enrolled in the study. All enrolled cases were divided into three groups namely $\mathrm{A}, \mathrm{B}$ and $\mathrm{C}$ based on treatment received like radiotherapy, chemotherapy and combination respectively and their effect on hearing was compared. Hearing acuity was assessed by doing PTA before and after completion of treatment and at 6 months follow up in every case.

\section{RESULTS}

In groups A, B and C SNHL was noted at higher frequencies of $4 \mathrm{kHz}$ and $8 \mathrm{kHz}$ during $1^{\text {st }}$ as well as final follow up. Hearing loss was found maximum in group $\mathrm{C}$ receiving combination treatment compared to the other two groups receiving in isolation. Hearing loss was the least in Group - A cases that received radiotherapy using the IMRT technique.

\section{CONCLUSIONS}

The possibility of SNHL is increased in cases receiving a combination of radio and chemotherapy (94\%). Extra care of shielding the cochlea is essentially required during treatment with high doses (> 60Gy) using conformal radiotherapy to limit the resultant radiotherapy-induced SNHL. Radiation-induced SNHL in the IMRT technique was the least (28 \%) in the group - A cases and hence should be employed in every case. Future searches for cases of head and neck malignancy the newer effective combination of chemotherapeutic drug and radiation obviating the ototoxicity needs to be continued.

\section{KEY WORDS}

Cisplatin, Radiotherapy, Intensity - Modulated, Audiometry, Pure - Tone, Ototoxicity, Hearing Loss, Sensorineural
Corresponding Author:

Dr. Abhay D. Havle,

Professor and Head,

Department of ENT,

Krishna Institute of Medical

Sciences "Deemed to Be" University,

Karad, Maharashtra, India.

E-mail: entpubkimsu@gmail.com

DOI: $10.14260 /$ jemds/2021/559

How to Cite This Article:

Prabhune SC, Havle AD, Shedge SA, et al. Sensorineural hearing loss as a sequelae of radiotherapy and chemotherapy in head and neck cancer - an observational study from Maharashtra, India. J Evolution Med Dent Sci 2021;10(33):2740-2744, DOI: $10.14260 /$ jemds $/ 2021 / 559$

Submission 16-03-2021,

Peer Review 29-05-2021,

Acceptance 05-06-2021,

Published 16-08-2021.

Copyright (C) 2021 Sanket Chandrashekhar Prabhune et al. This is an open access article distributed under Creative Commons Attribution License [Attribution 4.0 International (CC BY 4.0)] 


\section{BACKGROUND}

Radiotherapy is one of the treatment modalities for head and neck cancer. It is used either in the definitive setting or in the adjuvant setting after surgery whenever indicated. The auditory apparatus particularly the neuroepithelium present in the cochlea is sensitive to ionizing radiation and chemotherapy. This is because they remain in the field of irradiation and are also likely to get affected by chemotherapy which may lead to sensorineural hearing loss (SNHL). ${ }^{1}$ Health-related quality of life (HR - QOL) presents an important role in head and neck cancer cases. This is because they may have obvious debilitating problems of swallowing, speech, and hearing, as well as the psychological effects of loss of function and change in body image. The top three head and neck functional impairments (HNFI) mentioned by nasopharyngeal carcinoma survivors are problems with salivation (71\%), hearing (51\%), and swallowing (45\%). ${ }^{2}$ Advent of newer technologies like intensity modulated radiotherapy (IMRT) help us in giving dose constraints to sensitive organs and more or less can be achieved. Various published data clearly states that the dose to the cochlea can be significantly reduced by technologies like IMRT and prevent the development of SNHL even when concurrent cisplatin is used. ${ }^{3}$

Cisplatin (cis - diamminedichloroplatinum) is extensively used as a radiosensitiser in radiotherapy. It is also used as a chemotherapeutic agent in some head \& neck cancers like nasopharyngeal carcinoma. Cisplatin is also known to cause ototoxicity, particularly for higher frequencies. Platinuminduced and aminoglycoside-induced ototoxicity is characterized by the production of toxic levels of reactive oxygen species within the cochlea, with resultant destruction of cochlear hair cells leading to damage of the stria vascularis and spiral ganglion cells. Cochlear hair cell damage is generally dose-dependent, bilateral, and irreversible. In young children, the implications of even mild to moderate high-frequency hearing loss are particularly detrimental because they are generally in the process of acquiring language and communication skills at the time the hearing loss occurs, and therefore are dependent on the highfrequency fricative sounds (e.g., "the," "s") that are critical for speech discrimination, which may be unintelligible to a child with high-frequency hearing loss.

Once the cochlear sensory hair cells are destroyed, they cannot regenerate; therefore, drug-related sensorineural hearing loss is almost always bilateral and irreversible and also can be accompanied by tinnitus and vertigo. ${ }^{2}$ Since both agents seem to affect primarily the organ of Corti in the cochlea, and both modalities have their most pronounced effect in the high-frequency area, there has been some interest in determining their additive ototoxicity. ${ }^{3}$

Hearing sensitivity is typically described in a term of how loud the sound must be in order to be detected which is measured in decibels relative to the hearing of people without ear problems. Sensitivity is tested for more than one frequency. Sound frequencies are reported in cycles per second or Hertz (Hz). Though human beings can typically hear up to $20,000 \mathrm{~Hz}$, testing is usually done from 250 to $8,000 \mathrm{~Hz}$. Audiometry is a technique used by an audiologist or an otolaryngologist to measure hearing. There are two components of hearing loss - (a) conductive hearing loss, which is usually due to effects of radiation on the external and middle ears; (b) sensorineural hearing loss, which is related to radiation and chemotherapy effects on the cochlear and retro cochlear region. ${ }^{4}$

\section{Objectives}

- To determine and compare sensorineural hearing loss amongst cases of head and neck cancer treated by radiotherapy, chemotherapy either alone or in a combination of both.

- To evaluate the effects of chemoradiation amongst the cases of head and neck cancer coming from rural western Maharashtra.

\section{METHODS}

This prospective observational study was conducted from November 2018 to April 2020 at a tertiary care teaching hospital in rural western Maharashtra. After obtaining a clearance from the institutional ethics committee and obtaining the number of new biopsy proven cases requiring treatment every month which was 80 per 1000 population as in the pilot study, a total of 84 biopsy proven cases of head and neck cancer requiring radiotherapy and / or chemotherapy and having a normal hearing on PTA were enrolled in the study as per set inclusion criteria. Before receiving radiotherapy and or chemotherapy in all cases the name, age, sex of the patient, type of cancer, plan of treatment (radiotherapy / chemotherapy / chemo or combination of both), dose of radio and chemotherapy received were noted. Every patient was evaluated using proper history and clinical examination including, general examination and otoscopy and pure tone audiometry (PTA). All cases were divided into three groups according to the treatment they received as follows-

- Group A - Patients receiving radiotherapy.

- Group B - Patients receiving chemotherapy.

- Group C - Patients receiving a combination of both.

The pure tone audiometry was done before receiving radiotherapy and / or chemotherapy. (Baseline PTA). The first follow up PTA was done just after receiving radiotherapy and / or chemotherapy. Final follow up PTA was done after 6 months of completion of radiotherapy and / or chemotherapy. Most of the patients received conformal technique radiotherapy using a linear accelerator (3D - CRT) and IMRT and some received conventional RT. While either a chemotherapeutic drug like cisplatin, 5 - FU, Imatinib, paclitaxel, carboplatin and adriamycin was administered to the patient as per dose required. Hearing loss was assessed in each group at the time of first follow up and final follow up for comparing with baseline PTA. Quantification of hearing loss was done according to the American speech-languagehearing Association (ASHA). ${ }^{5}$

\section{Statistical Analysis}

Analysis of variance (ANOVA) test was used to find the significance of study parameters between three or more 
groups of patients for different frequencies with different time intervals and also for comparing three individual groups. Student $t$ test (two-tailed, independent) was used to find the significance of study parameters on a continuous scale between two groups intergroup analysis on metric parameters. The following assumptions on data were made, Assumptions: 1-Dependent variables should be normally distributed, 2- Samples were drawn from the population should be random and cases of the samples should be independent.

\section{RESULTS}

In the present observational study of 84 cases that received radiotherapy, chemotherapy and a combination of both for head and neck cancers the hearing loss was determined before and after treatment. It was further compared after treatment in all cases. Because of the limited period for the study, only 6 months follow up was considered after completion of treatment. Out of these, 6 cases were lost to follow up and the study was concluded with the remaining 78 cases. Out of these 84 cases 29, 22 and 33 were from the group - A (RT), B (CT) and C (RT - CT Combination) respectively. The $\mathrm{M}$ : $\mathrm{F}$ ratio amongst all groups was 80.95:19.05 (Table 1). The mean age amongst all groups was 49.7 years (Table 1 ). There was no significant difference in the mean age amongst individual groups.

\begin{tabular}{|c|c|c|c|c|}
\hline \multirow[b]{2}{*}{ Study Group } & \multicolumn{2}{|c|}{ No. of Patients } & \multirow{2}{*}{ Total } & \multirow{2}{*}{$\begin{array}{l}\text { Mean Age } \\
\text { (Years) }\end{array}$} \\
\hline & Males (\%) & Females (\%) & & \\
\hline Group A & $24(83)$ & $5(17)$ & $29(35 \%)$ & 48 \\
\hline Group B & $18(82)$ & $4(18)$ & $22(26 \%)$ & 51.41 \\
\hline Group C & $26(79)$ & $7(21)$ & $33(39 \%)$ & 49.7 \\
\hline Total & $68(80.95)$ & $16(19.05)$ & $84(100)$ & $49.70 \pm 8.55$ \\
\hline \multicolumn{5}{|c|}{ Table 1. Demographic Data } \\
\hline
\end{tabular}

The various primary tumours included in the study were of head and neck (hypopharynx, oral cavity, oropharynx, nasopharynx, paranasal tumours, parotid tumours, PNS etc.) and primary brain tumours where the inner ear was in the field of irradiation (Table 2). Planned radiotherapy treatment was executed with 3DCRT and IMRT on 6MV linear accelerator while the conventional technique was executed by telecobalt machine. $47(75.8 \%)$ patients were treated with 3DCRT technique, 8 (12.9\%) patients were treated with conventional technique and $7(11.3 \%)$ patients are treated with IMRT.

All patients treated by conventional technique received radiotherapy dose by more than 60 Grays at completion of treatment. Total of 62 cases received radiotherapy in group A and C. Concurrent cisplatin chemotherapy was received by 33 cases in group - C, while 22 cases from group B received only chemotherapy.

\begin{tabular}{|cccc|}
\hline Malignancy Site & $\begin{array}{c}\text { A Group } \\
\text { Frequency (\%) }\end{array}$ & $\begin{array}{c}\text { B Group } \\
\text { Frequency (\%) }\end{array}$ & $\begin{array}{c}\text { C Group } \\
\text { Frequency (\%) }\end{array}$ \\
Brain & $1(3.4)$ & NA & $1(3)$ \\
Hypopharynx & $9(31)$ & $4(18.2)$ & $14(42.4)$ \\
Laryax & $7(24.1)$ & $4(18.2)$ & $6(18.2)$ \\
Nasopharynx & $1(3.4)$ & $3(13.6)$ & $3(9.1)$ \\
Oral cavity & $5(17.2)$ & $4(18.2)$ & $1(3)$ \\
Oropharynx & $4(13.8)$ & $1(4.5)$ & $4(12.1)$ \\
PNS & $1(3.4)$ & $1(4.5)$ & $3(9.1)$ \\
NHL right tonsil & NA & $1(4.5)$ & NA \\
Parotid & NA & $4(18.2)$ & $1(3)$ \\
\hline \multicolumn{4}{c}{ Table2. Distribution of Cancer Site } \\
\hline \multicolumn{3}{c}{} \\
\hline \multicolumn{4}{c}{}
\end{tabular}

All patients in group - $\mathrm{C}$ received concurrent cisplatin chemotherapy with the cumulative dose of $220 \mathrm{mg}-730 \mathrm{mg}$. While patients in Group B received either of chemotherapy drug like injection cisplatin (220 mg - $730 \mathrm{mg}$ ), injection 5FU (30 mg - $180 \mathrm{mg}$ ), Tab imatinib (400 mg - $800 \mathrm{mg}$ ), injection paclitaxel (150 mg - $265 \mathrm{mg}$ ), injection carboplatin (130 mg - $300 \mathrm{mg}$ ) and injection Adriamycin (65 mg - 210 $\mathrm{mg}$ ) to dose required (Table 3 ).

\begin{tabular}{|c|c|c|c|}
\hline & Technique & No. of Cases & $(\%)$ \\
\hline \multirow{3}{*}{$\begin{array}{l}\text { Radiotherapy } \\
\text { (RT) }\end{array}$} & Conventional & 8 & 9.52 \\
\hline & 3D - CRT & 14 & 16.67 \\
\hline & IMRT & 7 & 8.33 \\
\hline $\begin{array}{l}\text { Combination } \\
\text { Therapy }\end{array}$ & $\begin{array}{c}\text { 3D - CRT + Inj. Cisplatin (220 mg - } 730 \\
\text { mg) }\end{array}$ & 33 & 39.28 \\
\hline \multirow{6}{*}{$\begin{array}{l}\text { Chemotherapy } \\
\text { (CT) }\end{array}$} & Inj 5 - FU (30mg - $180 \mathrm{mg})$ & 1 & 7.14 \\
\hline & Tab Imatinib (400mg - $800 \mathrm{mg}$ ), Inj. & 3 & 3.57 \\
\hline & Paclitaxel (150mg - $265 \mathrm{mg})$ & 4 & 4.76 \\
\hline & Inj. Carboplatin (130 mg - 300mg), & 6 & 7.14 \\
\hline & Adriamycin (65mg - $210 \mathrm{mg})$,Inj. & 2 & 2.38 \\
\hline & Inj. Cisplatin(220mg - $730 \mathrm{mg})$ & 6 & 7.14 \\
\hline Total & & 84 & 100 \\
\hline
\end{tabular}

In group - A it was observed that there was a significant $(\mathrm{P}<0.0001)$ decrease in hearing at $1^{\text {st }}$ and final follow up compared to baseline PTA especially at higher frequencies (Table 4). Also, in the group - A (RT) wherein three techniques of delivering radiotherapy were used like conventional RT in 8 cases, conformal RT like 3DCRT in 14 and IMRT in 7.

In these 8 cases who received conventional technique, 7 had mild SNHL and 1 had moderate SNHL, similarly in 14 who received 3DCRT all had mild SNHL and those in 7 who received IMRT technique only 2 had mild SNHL. Similarly, in group - B it was observed that there was a significant $\mathrm{P}<$ 0.0001 ) decrease in hearing at $1^{\text {st }}$ and final follow up as compared to baseline PTA especially at higher frequencies (Table 4). While, in the group - C we noted that there was a higher degree of hearing loss compared with the group - A and B especially at higher frequencies and it was found statistically significant with $\mathrm{P}<0.0001$ (Table 4).

\begin{tabular}{|c|c|c|c|c|c|c|c|c|c|}
\hline & & $250 \mathrm{hz}$ & $500 \mathrm{hz}$ & $1000 \mathrm{hz}$ & $2000 \mathrm{hz}$ & $4000 \mathrm{hz}$ & $8000 \mathrm{hz}$ & F - Value & P - Value \\
\hline \multirow{3}{*}{ Group A: } & Baseline (PTA (N = 58 & $15.24 \pm 3.2$ & $14.75 \pm 3.42$ & $14.82 \pm 3.63$ & $15.71 \pm 3.42$ & $15.08 \pm 3.47$ & $15.08 \pm 3.47$ & 1.237 & 0.3 \\
\hline & PTA at $1^{\text {st }}$ follow up $(\mathrm{N}=54) \#$ & $16.06 \pm 3.8$ & $15.56 \pm 2.8$ & $17.17 \pm 3.08$ & $16.96 \pm 2.6$ & $23.63 \pm 2.87$ & $31.11 \pm 5.98$ & 148.04 & $<0.0001^{*}$ \\
\hline & PTA at final follow up $(\mathrm{N}=52) \#$ & $15.90 \pm 3.82$ & $15.45 \pm 2.74$ & $17.09 \pm 3.12$ & $17.57 \pm 2.09$ & $25.31 \pm 3.05$ & $36.27 \pm 4.76$ & 306.71 & $<0.0001^{*}$ \\
\hline \multirow{3}{*}{ Group B: } & Baseline PTA $(\mathrm{N}=44)$ & $15.18 \pm 2.63$ & $15.18 \pm 2.8$ & $15.11 \pm 2.8$ & $15.91 \pm 2.46$ & $15.51 \pm 2.75$ & $15.18 \pm 2.63$ & 0.59 & 0.71 \\
\hline & PTA at $1^{\text {st }}$ follow up $(\mathrm{N}=42)$ & $15.48 \pm 1.84$ & $15.76 \pm 2.6$ & $15.50 \pm 2.78$ & $16.64 \pm 2.9$ & $22.91 \pm 3.4$ & $30.5 \pm 4.83$ & 154.03 & $<0.0001^{*}$ \\
\hline & PTA at final follow up $(n=42)$ & $16.48 \pm 1.94$ & $16.76 \pm 2.65$ & $15.40 \pm 2.99$ & $17.54 \pm 2.86$ & $23.76 \pm 3.1$ & $32.96 \pm 4.96$ & 160.2 & $<0.0001^{*}$ \\
\hline \multirow{3}{*}{ Group C: } & Baseline PTA (N = 66) & $15.34 \pm 3.17$ & $14.95 \pm 3.36$ & $15.17 \pm 2.73$ & $14.83 \pm 2.9$ & $18.80 \pm 2.91$ & $15.34 \pm 3.17$ & 0.47 & 0.8 \\
\hline & PTA at $1^{\text {stfollow up }(\mathrm{N}=62)}$ & $15.55 \pm 2.52$ & $15.96 \pm 3.63$ & $16.6 \pm 2.72$ & $16.76 \pm 3.03$ & $25.39 \pm 2.8$ & $39.45 \pm 4.92$ & 240.37 & $<0.0001^{*}$ \\
\hline & PTA at final follow up $(\mathrm{N}=62)$ & $15.57 \pm 2.5$ & $15.99 \pm 3.66$ & $16.8 \pm 2.81$ & $16.78 \pm 3.14$ & $25.87 \pm 2.96$ & $42.03 \pm 4.57$ & 259.61 & $<0.0001^{*}$ \\
\hline \multicolumn{10}{|c|}{ Table 4. Group Wise PTA during Follow Up } \\
\hline
\end{tabular}




\section{DISCUSSION}

Out of 84 initially enrolled cases, in 78 the observations were made during six months follow up and then concluded. The primary site of the malignancy was of little interest during interpretation of the results except that oral cavity primaries were less likely to result in sensorineural hearing loss than the other head and neck areas as they remained away from the cochlea. The majority of the available studies about SNHL are with nasopharyngeal carcinoma. ${ }^{3,4,6,7,8}$ The present study included various head and neck primaries and also the tumours of the brain (primary brain tumours $3.4 \%$, hypopharynx $31 \%$, oral cavity $17.2 \%$, nasopharynx $3.4 \%$, oropharynx $13.8 \%$, PNS $3.4 \%$, parotid $3 \%$ ) whereas inner ear remained in the field of irradiation. A similar type of recent study by Zuur et al. having a distribution of cases not confining to nasopharynx alone is available. ${ }^{9}$

The radiation effects and their doses on the inner ear are studied on various age groups in the present study. The median age of the present study was 51 years (Mean \pm SD: $49.70 \pm 8.55$ ). Studies done by Bhandare et al. also stated that the chemoradiation affected hearing, especially in the elderly age group (Mean age 53 years). ${ }^{6}$ Merchant et al. studied the effects of 3DCRT on children with a median age of 9.5 years. ${ }^{10}$

A hearing decrement of $\geq 10 \mathrm{~dB}$ was considered clinically significant in the present study as reported by Pan et al. ${ }^{9}$ According to this criterion, in cases receiving radiotherapy in group A $24.1 \%$ and $68.9 \%$ had significant hearing loss for 4 $\mathrm{kHz}$ and $8 \mathrm{kHz}$ respectively after treatment; $31.03 \%$ and 82.7 $\%$ patients had significant hearing loss for $4 \mathrm{kHz}$ and $8 \mathrm{kHz}$ respectively at 6 months follow-up.

Similarly, in cases receiving only chemotherapy in the group - B, $31.8 \%$ and $81.8 \%$ patients had significant hearing loss at $4 \mathrm{kHz}$ and $8 \mathrm{kHz}$ respectively after treatment, $36.3 \%$ and $86.3 \%$ had significant hearing loss for $4 \mathrm{kHz}$ and $8 \mathrm{kHz}$ respectively at 6 months follow-up. Similarly, in patients receiving combination - RTCT in group C, $51.5 \%$ and $87.1 \%$ patients had significant hearing loss for $4 \mathrm{kHz}$ and $8 \mathrm{kHz}$ respectively after treatment; $57.5 \%$ and $90 \%$ patients had significant hearing loss for $4 \mathrm{kHz}$ and $8 \mathrm{kHz}$ respectively at follow-up. Our incidence is higher compared to the literature, but the number is too less due to limited time. The incidence of post-RT sensorineural deficit has been reported to range from $0 \%$ to $50 \%$. Kwong et al. reported a $24 \%$ incidence of sensorineural hearing loss particularly for higher frequencies, following radical radiotherapy for nasopharyngeal carcinoma. ${ }^{11}$ Bhandare et al. observed SNHL in $15.1 \%$ of patients. Merchant et al. found that the rate of permanent hearing loss ranged from $24.2 \%$ to $36 \%$ for doses approaching $60 \mathrm{~Gy} .{ }^{10}$ In this study we observed, the cases that received radiotherapy in group - A, incidence of SNHL was least in patients who received radiation through IMRT technique which was in accordance with the studies done by Eleonoor A. R Theunissen et al. and Zuur et al. where the patients receiving radiation through IMRT had the least incidence of SNHL. ${ }^{3,9}$ Zuur et al. in his prospective analysis of hearing loss due to concurrent daily low dose cisplatin chemoradiation for locally advanced head and neck cancers reported a total incidence of ototoxicity in CTCAEV3.0 as 31 $\%$ in audiograms up to $8 \mathrm{kHz} .{ }^{9}$ Low dose cisplatin chemo irradiation (CRT) caused less acute hearing loss (31\%), compared to high dose cisplatin CRT (78\%). Chan et al. in their longitudinal study on SNHL after treatment of nasopharyngeal carcinoma found that RT alone and chemoradiotherapy resulted in $40 \%$ and $56.4 \%$ persistent SNHL, respectively, after a median follow-up of 2 years. ${ }^{12}$ In the above study by Chan SNHL at a high frequency was more frequent in the chemoradiotherapy group than in the RT alone group (55\% VS $33.3 \%$ ), but not at a low frequency which was in this study too. ${ }^{1}$ In this study, there was significant SNHL at higher frequencies especially at $4 \mathrm{kHz}$ and $8 \mathrm{kHz}$ in patients receiving only chemotherapy $(\mathrm{P}<0.0001)$.

In this study, around $31.03 \%$ of cases receiving radiotherapy in the group - A had SNHL at 6 months follow up, similarly $90.1 \%$ of cases receiving concurrent chemoradiation in the group - C had SNHL at 6 months follow up period. These findings were in accordance with the study done by Mahdavi SR et al. that says $84 \%$ of cases had SNHL who received chemoradiation and $26 \%$ of cases had SNHL who received only radiotherapy. ${ }^{7}$ Similarly study done by Monika Patel PS et al. $45.45 \%$ of cases had SNHL who received radiotherapy and $70.58 \%$ of cases had SNHL who received concurrent chemoradiation. ${ }^{1}$ In study done by Raies Ahmad Begh et al. $65 \%$ of cases had SNHL who received concurrent chemoradiotherapy. ${ }^{13}$

In this study, the total dose of radiation given was > 60Gy to all cases in the group - A and C. At the time of final follow up the hearing found in group - $\mathrm{A}$ at $2 \mathrm{kHz}, 4 \mathrm{kHz}$ and $8 \mathrm{kHz}$ was significantly affected (P-value 0.001, < 0.0001 and < 0.0001 ) respectively. In group - $\mathrm{C}$ there was similar significant hearing loss found at $1 \mathrm{kHz}, 2 \mathrm{kHz}, 4 \mathrm{kHz}$, and 8 $\mathrm{kHz}$ (P value $0.0036,0.0006,<0.0001,<0.0001$ ) respectively. SNHL is found to occur after higher doses of RT according to various studies, however, the total dose of RT also varies. Grau et al. found in their study greater SNHL for doses $>50$ Gy. ${ }^{14}$ Pan et al. in a prospective study showed SNHL for doses $\geq 45$ Gy. ${ }^{11}$ Chan et al. reported that mean threshold of 48 Gy was statistically significant in predicting SNHL. ${ }^{12}$ Petsuksiri et al. reported that mean dose $\leq 50 \mathrm{~Gy}$ appeared to have lower incidences of SNHL and the incidences of SNHL tended to be higher if they received a mean radiation dose of $>50 \mathrm{~Gy} .{ }^{15}$

SNHL was considered as a late complication following radiotherapy. In this study SNHL was noted for $8000 \mathrm{~Hz}$ at the completion of treatment and at 6 months follow up. And for $4000 \mathrm{~Hz}$ SNHL was noted only at 6 months. The onset of SNHL following radiotherapy was noted for higher frequencies and at longer periods during follow up. ${ }^{10,12,14}$ Monica Patel PS et al. in their study observed that there was significant hearing loss in both groups RT and CRT after one month of chemoradiation. ${ }^{1}$ Bhandare et al. reported the median interval between RT and the development of persistent SNHL at 1.8 years (range, $0.5-5.9$ years). ${ }^{10} \mathrm{Grau}$ et al. and Chan et al. reported that most of SNHL was first noted 12 months after the RT completion. ${ }^{14,12}$ Pan et al. did not find any time association between hearing loss and RT.11 The present study with 6 months follow up period may be less and require longer follow up after chemoradiation to conclude SNHL. Concurrent chemoradiation has become the standard of care for the majority of head and neck cancers. Cisplatin is the most commonly used drug for radiosensitization. There are various published data suggesting the increased ototoxicity when both radiation and cisplatin chemotherapy are used concurrently. ${ }^{3,4,7,8,1}$ In the 
present study concurrent use of cisplatin chemotherapy has increased the SNHL for $2000 \mathrm{~Hz}, 4000 \mathrm{~Hz}$, and $8000 \mathrm{~Hz}$ by the end of treatment. However, the SNHL has recovered for 2000 $\mathrm{Hz}$ and at 6 months follow up and persisted for $4000 \mathrm{~Hz}$ and $8000 \mathrm{~Hz}$.

The cumulative dose of cisplatin is an important factor for SNHL. Mahdavi SR et al. reported that hearing loss after cisplatin therapy occurs mainly at high frequencies and cisplatin dosages more than $60 \mathrm{mg} / \mathrm{ml}$. This risk increased by almost 3 - fold, at cumulative doses of $701-1300 \mathrm{mg} /$ ml. ${ }^{7,1}$ Total cumulative dose of cisplatin received in this study group - $\mathrm{C}$ that is the patients receiving concurrent chemoradiation ranged from 220 to $730 \mathrm{mg}$. Significant SNHL was observed at $4 \mathrm{kHz}$ and $8 \mathrm{kHz}$ during the first follow-up and it persisted up to final follow-up as patients in this study received moderate to a high dose of cisplatin.

\section{CONCLUSIONS}

The possibility of SNHL is increased in cases receiving a combination of radio and chemotherapy (94\%). Therefore, extra care of shielding the cochlea is essentially required during treatment with high doses (> 60Gy) while using conformal radiotherapy to limit the resultant radiotherapyinduced SNHL. IMRT technique used in the group - A cases resulted in the least (28\%) radiation-induced SNHL and hence should be employed in every case. Future search for newer effective chemotherapeutic drugs and their combination with radiation technologies that will obviate the ototoxicity in patients with head and neck malignancies need to be continued.

Data sharing statement provided by the authors is available with the full text of this article at jemds.com.

Financial or other competing interests: None.

Disclosure forms provided by the authors are available with the full text of this article at jemds.com.

\section{REFERENCES}

[1] Patel M. Prospective study of sensorineural hearing loss in patients of head and neck cancers after radiotherapy and chemotherapy. J Otolaryngol ENT Res 2018;10(4):207-11.

[2] Fang FM, Chiu HC, Kuo WR, et al. Health-related quality of life for nasopharyngeal carcinoma patients with cancer-free survival after treatment. Int J Radiation Oncology Biol Phys 2002;53(4):959-68.

[3] Theunissen EAR. Sensorineural hearing loss in patients with head and neck cancer after chemoradiotherapy and radiotherapy: a systematic review of the literature. Head Neck 2015;37(2):281-92.

[4] Jain A, Banerjee PK, Manjunath D. Effects of chemoradiation on hearing in patients with head and neck malignancies: experience at a tertiary referral care hospital. Indian J Otolaryngol Head Neck Surg 2016;68(4):1-6.

[5] American Speech-Language-Hearing Association. Classification of speech-language pathology and audiology procedures and communication disorders. ASHA 1987;29(12):49-53.

[6] Linskey ME, Johnstone PAS. Radiation tolerance of normal temporal bone structures: implications for gamma knife stereotactic radiosurgery. Int J Radiat Oncol Biol Phys 2003;57(1):196-200.

[7] Mahdavi SR, Rezaeyan A, Nikoofar A, et al. Comparison of radiation and chemoradiation induced sensorineural hearing loss in head and neck cancer patients. J Cancer Res Ther 2020;16(3):539-45.

[8] Bhandare N, Jackson A, Eisbruch A, et al. Radiation therapy and hearing loss. Int J Radiat Oncol Biol Phys 2010;76(3 Suppl):S50-7.

[9] Zuur CL, Simis YJ, Lamers EA, et al. Risk factors for hearing loss in patients treated with intensity-modulated radiotherapy for head-and-neck tumors. Int J Radiat Oncol Biol Phys 2009;74(2):490-6.

[10] Merchant TE, Gould CJ, Xiong X, et al. Early neurootologic effects of three-dimensional irradiation in children with primary brain tumors. Int J Radiat Oncol Biol Phys 2004;58(4):1194-207.

[11] Kwong DLW, Wei WI, Sham JST, et al. Sensorineural hearing loss in patients treated for nasopharyngeal carcinoma: a prospective study of the effect of radiation and cisplatin treatment. Int J Radiat Oncol Biol Phys 1996;36(2):281-9.

[12] Chan SH, Ng WT, Kam KL, et al. Sensorineural hearing loss after treatment of nasopharyngeal carcinoma: a longitudinal analysis. Int J Radiat Oncol Biol Phys 2009;73(5):1335-42.

[13] Begh RA, Kaul D, Saraf A, et al. Hearing loss in patients with head and neck cancer post chemoradiotherapy. International Journal of Otorhinolaryngology and Head and Neck Surgery 2020;6(5):830-34.

[14] Grau C, Overgaard J. Postirradiation sensorineural hearing loss: a common but ignored late radiation complication. Int J Radiat Oncol Biol Phys 1996;36(2):515-7.

[15] Petsuksiri J, Sermsree A, Thephamongkhol K, et al. Sensorineural hearing loss after concurrent chemoradiotherapy in nasopharyngeal cancer patients. Radiat Oncol 2011;6:19. 